16

\title{
Инварианты коэффициента отражения
}

\author{
() Л.А. Федюхин ${ }^{1}$, А.В. Горчаков ${ }^{2}$, Е.А. Колосовский ${ }^{1}$ \\ ${ }^{1}$ Институт ффизики полупроводников Сибирского отделения РАН, \\ 630090 Новосибирск, Россия \\ ${ }^{2}$ Новосибирский государственный университет, \\ 630090 Новосибирск, Россия \\ e-mail: leogal2007@mail.ru
}

Поступила в редакцию 21.06.2019 г.

В окончательной редакции 24.10.2019 г.

Принята к публикации 01.11.2019 г.

\begin{abstract}
Детально проанализирован коэффициент отражения плоской монохроматической волны, линейно поляризованной в плоскости падения, от трехслойной структуры. Решена обратная задача эллипсометрии для трехслойной структуры. Показано существование двух инвариантов коэффициента отражения для плоскопараллельной пластины. Предложен набор трех измеряемых параметров, не используемых ранее, позволяющий восстановить материальные параметры слоя. Получены аналитические выражения как для коэффициента отражения и углов падения для ряда важных частных случаев, так и для прямого расчета диэлектрической проницаемости и толщины слоя по измеренным значениям наблюдаемых параметров.
\end{abstract}

Ключевые слова: инвариант коэффициента отражения, обратная задача эллипсометрии, угол Брюстера, тонкие пленки.

DOI: $10.21883 /$ OS.2020.02.48975.219-19

\section{Введение}

Вопросам определения параметров тонких пленок, осажденных на поверхности кристаллов, посвящено весьма большое количество как теоретических $[1,2]$, так и экспериментальных $[2,3]$ работ, что обусловлено важностью темы для разработки и модернизации технологии получения пленок с заранее заданными свойствами. Как правило, для описания оптических характеристик тонких пленок на поверхности кристаллов используются варианты многослойной модели, суть которой заключается в моделировании формы профиля показателя преломления пленки, начиная от ступенчатой и заканчивая произвольной [4]. Используя далее формулы Френеля, авторы рассчитывают коэффициенты отражения/прохождения при падении электромагнитной волны на поверхность такой структуры и подбирают параметры выбранной модели, добиваясь наилучшего соответствия с наблюдаемыми величинами. В качестве наблюдаемых параметров традиционно используют интенсивность падающего излучения и угол падения. Широкое распространение получили эллипсометрические параметры $\psi$ и $\Delta$. В [5] предложено контролировать рост тонких пленок методом угловой рефлектометрии в области угла Брюстера, а для оптической характеристики объектов и, в частности, для анализа тонкопленочных структур предложено использовать производную по углу от логарифма измеренных интенсивностей отражения от пленки при различных длинах волн зондирующего излучения.

Несмотря на серьезные успехи подобного моделирования [2,4], вопрос выбора наблюдаемых параметров, позволяющих однозначно восстановить материальные параметры структур, до конца не решен.

\section{Анализ коэффициента отражения вблизи угла Брюстера}

Амплитудный коэффициент отражения плоской монохроматической электромагнитной волны, линейно поляризованной в плоскости падения, от трехслойной структуры (рис. 1), представляющей собой полубесконечную среду с диэлектрической проницаемостью $\varepsilon_{1}$, слой толщиной $d$ с диэлектрической проницаемостью $\varepsilon_{\mathrm{tr}}$, и еще одну полубесконечную среду с диэлектрической проницаемостью $\varepsilon_{2}$, может быть выражен через коэффициенты отражения от полубесконечных сред (коэффициенты

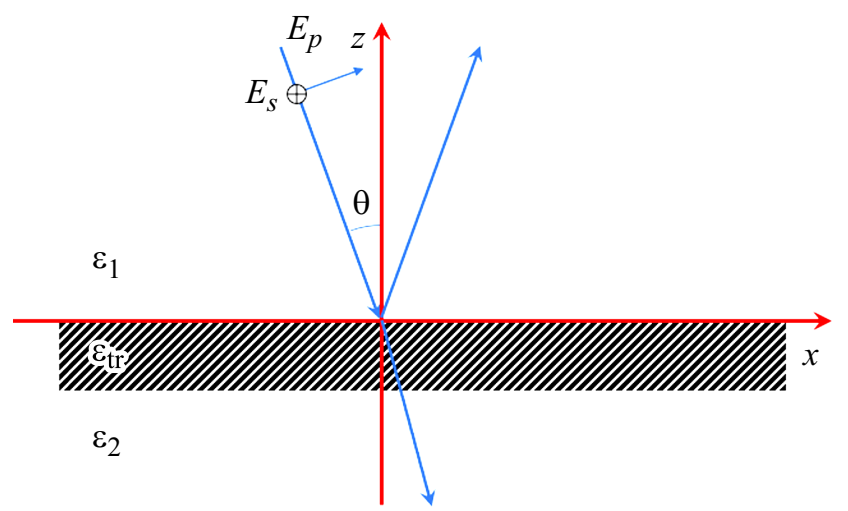

Рис. 1. Схема слоистой структуры. 
Френеля) [6]:

$$
r=\frac{r_{1 \mathrm{tr}}+r_{\mathrm{tr} 2} \exp (-2 i \delta)}{1+r_{1 \mathrm{tr}} r_{\operatorname{tr} 2} \exp (-2 i \delta)}
$$

Здесь

$$
\begin{aligned}
& r_{1 t r}=\frac{p_{1}-p_{\mathrm{tr}}}{p_{1}+p_{\mathrm{tr}}}, \quad r_{\mathrm{tr} 2}=\frac{p_{\mathrm{tr}}-p_{2}}{p_{\mathrm{tr}}+p_{2}}, \quad p_{1}=\frac{\cos \theta}{\sqrt{\varepsilon_{1}}} \\
& p_{2}=\frac{\sqrt{\varepsilon_{2}-\varepsilon_{1} \sin ^{2} \theta}}{\varepsilon_{2}}, \quad p_{\mathrm{tr}}=\frac{\sqrt{\varepsilon_{\mathrm{tr}}-\varepsilon_{1} \sin ^{2} \theta}}{\varepsilon_{\mathrm{tr}}}
\end{aligned}
$$

$\delta=2 \pi \frac{d}{\lambda} \sqrt{\varepsilon_{\mathrm{tr}}-\varepsilon_{1} \sin ^{2} \theta}-$ фазовая толщина слоя, $\lambda-$ длина волны падающего на структуру излучения, $\theta-$ угол падения волны на границу раздела сред, $\varepsilon_{1}, \varepsilon_{2}$, $\varepsilon_{\mathrm{tr}}$ - диэлектрические проницаемости внешней среды, подложки и слоя соответственно, $d-$ толщина слоя.

Рассматриваемая трехслойная структура является удобной моделью при решении обратной задачи - определения материальных параметров структуры по результатам измерений коэффициента отражения в различных экспериментальных геометриях. Именно это обстоятельство обусловливает постоянный интерес исследователей к анализу соотношений, определяемых (1).

В этой части настоящего сообщения мы акцентируем внимание на анализе зависимости квадрата модуля коэффициента отражения $R=|r|^{2}$ (далее коэффициента отражения) как от угла падения $\theta$, так и от толщины слоя вблизи отдельных точек, при которых $R$ имеет характерные особенности. В этих случаях удается получить простые аналитические выражения, позволяющие, в свою очередь, найти простые соотношения для определения материальных параметров структуры. Проанализируем ситуацию, при которой коэффициент Френеля $r_{1 \mathrm{tr}}=0$. Как следует из $(2)$, в этом случае

$$
\frac{p_{1}-p_{\mathrm{tr}}}{p_{1}+p_{\mathrm{tr}}}=0,
$$

что определяет угол падения, при котором это условие выполняется:

$$
\theta_{\mathrm{tr}}=\operatorname{arctg} \sqrt{\frac{\varepsilon_{\mathrm{tr}}}{\varepsilon_{1}}} .
$$

Значение коэффициента отражения в этом случае не зависит от толщины слоя $d$ и определяется только диэлектрическими проницаемостями подложки и слоя:

$$
\begin{aligned}
R_{\mathrm{tr}}=|r|^{2} & =\left|r_{\mathrm{tr} 2} \exp (-2 i \psi)\right|^{2}=\left|r_{\mathrm{tr} 2}\right|^{2} \\
& =\left|\frac{\varepsilon_{2}-\sqrt{\varepsilon_{2} \varepsilon_{\mathrm{tr}}+\varepsilon_{2} \varepsilon_{1}-\varepsilon_{1} \varepsilon_{\mathrm{tr}}}}{\varepsilon_{2}+\sqrt{\varepsilon_{2} \varepsilon_{\mathrm{tr}}+\varepsilon_{2} \varepsilon_{1}-\varepsilon_{1} \varepsilon_{\mathrm{tr}}}}\right|^{2} .
\end{aligned}
$$

В то же время фазовая толщина слоя $\delta$ по-прежнему изменяется пропорционально $d$ :

$$
\delta_{\mathrm{tr}}=2 \pi \frac{d}{\lambda} \operatorname{tg} \theta_{\mathrm{tr}} \sqrt{\varepsilon_{1}\left(2 \sin ^{2} \theta_{\mathrm{tr}}-1\right)}=2 \pi \frac{d}{\lambda} \sqrt{\frac{\varepsilon_{\mathrm{tr}} \varepsilon_{1}}{\varepsilon_{\mathrm{tr}}+\varepsilon_{1}}} .
$$

Соотношение (4) позволяет рассчитать диэлектрическую проницаемость слоя $\varepsilon_{\text {tr }}$ при известном значении диэлектрической проницаемости подложки $\varepsilon_{2}$ и измеренном экспериментально значении коэффициента отражения в точке, определяемой соотношением (3). Найти значение $\theta_{\text {tr }}$ можно графически, построив теоретическую зависимость коэффициента отражения вблизи угла Брюстера. Ее пересечение с экспериментальной зависимостью однозначно определяет угол $\theta_{\mathrm{tr}}$, а следовательно, и диэлектрическую проницаемость слоя.

Значение коэффициента отражения в точке $\theta_{\mathrm{tr}}$ существенно зависит от соотношений между материальными параметрами сред структуры. Нетрудно видеть, что при равенстве диэлектрических проницаемостей верхней и нижней сред $\left(\varepsilon_{1}=\varepsilon_{2}\right)$, что соответствует плоскопараллельной пластине, находящейся в воздухе, коэффициент отражения имеет глубокий минимум. Это позволяет с высокой точностью определить диэлектрическую проницаемость пластины, однако только в том случае, когда удается минимизировать изменения состава приповерхностного слоя образца. Противоположная ситуация, при которой коэффициент отражения близок к единице, наблюдается при значении диэлектрической проницаемости слоя

$$
\varepsilon_{\mathrm{tr}}=\frac{\varepsilon_{1} \varepsilon_{2}}{\varepsilon_{1}-\varepsilon_{2}}
$$

Другой характерной точкой зависимости коэффициента отражения от угла падения является ситуация, при которой коэффициент отражения строго обращается в нуль. Известно [7], что для линейно поляризованной плоской монохроматической электромагнитной волны, падающей на границу раздела двух сред с диэлектрическими проницаемостями $\varepsilon_{1}$ и $\varepsilon_{2}$, коэффициент отражения обращается в нуль при угле Брюстера:

$$
\theta_{\mathrm{br}}=\operatorname{arctg} \sqrt{\frac{\varepsilon_{2}}{\varepsilon_{1}}} .
$$

Для трехслойной структуры ситуация усложняется. На рис. 2 приведена зависимость коэффициента отражения вблизи угла Брюстера при различных значениях толщины слоя. По мере увеличения толщины значения углов падения, соответствующих минимуму коэффициента отражения, смещаются от значения угла Брюстера для двухслойной структуры в сторону больших значений угла падения вплоть до некоторого угла $\theta_{2}$. При дальнейшем увеличении толщины эти значения начинают смещаться в сторону меньших значений угла падения вновь до угла Брюстера, где опять меняют направление смещения. Такое осциллирующие поведение значения углов падения, соответствующих минимуму коэффициента отражения, отображено на рис. 3. Важно отметить, что значение коэффициента отражения в точках минимума строго равно нулю, а положение граничных значений $\theta_{\mathrm{br}}$ и $\theta_{2}$ не зависит от толщины слоя. В точках, где коэффициент отражения максимален, его значение определяется (4) и также не зависит от толщины слоя. 


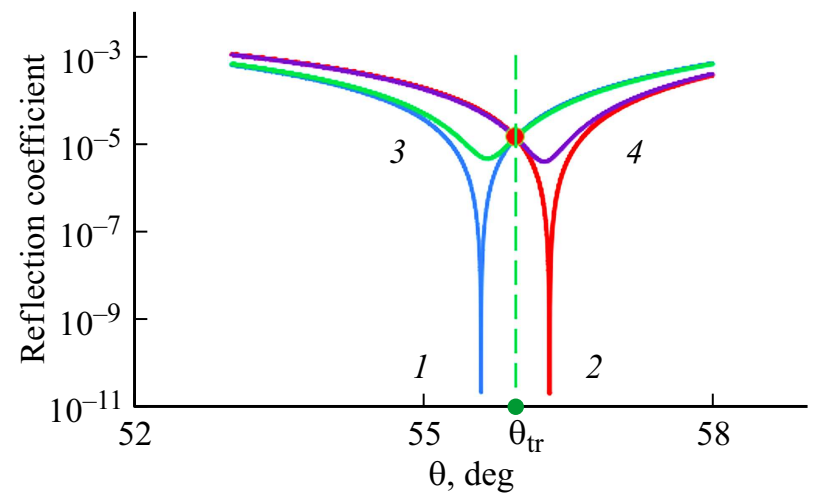

Рис. 2. Численный расчет зависимости коэффициента отражения от угла падения $\theta$ вблизи угла Брюстера при следующих параметрах структуры: $\lambda=532 \mathrm{~nm}, \varepsilon_{1}=1, \varepsilon_{2}=2.1316$, $\varepsilon_{\mathrm{tr}}=2.1904$. Расчет выполнен при четырех значениях толщины слоя $d$ : 0 (1), 109 (2), 21 (3), $89 \mathrm{~nm}(4)$.

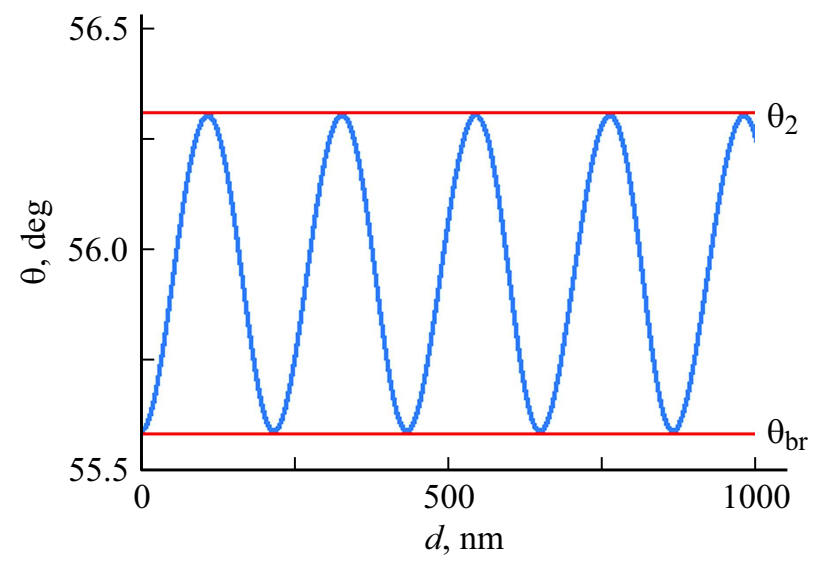

Рис. 3. Зависимость углов падения $\theta$, соответствующих минимуму коэффициента отражения, от толщины слоя $d$ в области $\theta_{\mathrm{br}}-\theta_{2}$.

Несмотря на то, что формула (1) является точным решением уравнений Максвелла для трехслойной структуры и полностью описывает поведение коэффициента отражения, для практического использования полезно получить более простые аналитические выражения для коэффициента отражения в области углов падения $\theta_{\mathrm{br}}-\theta_{2}$. Начать целесообразно с условий, при которых фазовая толщина слоя $\delta$ исключает зависимость коэффициента отражения от его толщины. А именно, проанализируем ситуацию, при которой $\delta=\pi / 2$. В этом случае

$$
R=|r|^{2}=\frac{\left[r_{1 \operatorname{tr}}\left(1+r_{\operatorname{tr} 2}^{2}\right) \pm r_{\operatorname{tr} 2}\left(1+r_{1 \mathrm{tr}}^{2}\right)\right]^{2}}{\left[1+r_{1 \operatorname{tr}}^{2} r_{\operatorname{tr} 2}^{2} \pm 2 r_{1 \mathrm{tr}} r_{\mathrm{tr} 2}\right]^{2}}
$$

Равенство нулю возможно при обращении в нуль числителя:

$$
r_{1 \mathrm{tr}}\left(1+r_{\mathrm{tr} 2}^{2}\right) \pm r_{\mathrm{tr} 2}\left(1+r_{1 \mathrm{tr}}^{2}\right)=0
$$

Нетрудно убедиться, что решением уравнения со знаком плюс является значение угла падения $\theta=\theta_{\mathrm{br}}$. Минимум достигается периодически, по мере того как фазовая толщина слоя становится равной $\pi n$. Толщина, при которой коэффициент отражения обращается в нуль:

$$
d=\frac{n \lambda}{2} \sqrt{\frac{\varepsilon_{2}+\varepsilon_{1}}{\varepsilon_{\mathrm{tr}} \varepsilon_{2}+\varepsilon_{\mathrm{tr}} \varepsilon_{1}-\varepsilon_{1} \varepsilon_{2}}},
$$

имеет особенность вблизи значения диэлектрической проницаемости слоя

$$
\varepsilon_{\mathrm{tr}}=\frac{\varepsilon_{1} \varepsilon_{2}}{\varepsilon_{1}+\varepsilon_{2}}
$$

В этой точке период осцилляций для угла падения $\theta=\theta_{\mathrm{br}}$ при изменении толщины слоя неограниченно возрастает.

Уравнение (7) со знаком минус, после подстановки выражений для $r_{1 \mathrm{tr}}$ и $r_{\text {tr2 }}$ из $(2)$, дает

$$
\left[\frac{p_{1}-p_{\mathrm{tr}}}{p_{1}+p_{\mathrm{tr}}}-\frac{p_{\mathrm{tr}}-p_{2}}{p_{\mathrm{tr}}+p_{2}}\right]\left[1-\frac{p_{1}-p_{\mathrm{tr}}}{p_{1}+p_{\mathrm{tr}}} \frac{p_{\mathrm{tr}}-p_{2}}{p_{\mathrm{tr}}+p_{2}}\right]=0 .
$$

Равенство нулю возможно, когда хотя бы один из множителей равен нулю, что равносильно условию

$$
p_{1} p_{2}= \pm p_{\mathrm{tr}}^{2}
$$

или, подставляя значения $p_{1}, p_{2}, p_{\mathrm{tr}}$, из (2),

$$
\frac{\varepsilon_{\mathrm{tr}}-\varepsilon_{1} \sin ^{2} \theta}{\varepsilon_{\mathrm{tr}}^{2}}= \pm \frac{\cos \theta}{\sqrt{\varepsilon_{1}}} \frac{\sqrt{\varepsilon_{2}-\varepsilon_{1} \sin ^{2} \theta}}{\varepsilon_{2}} .
$$

Уравнения (7) позволяют, с одной стороны, определить углы, при которых коэффициент отражения при определенных значениях толщины слоя строго обращается в нуль:

$$
\left\{\begin{aligned}
\operatorname{tg} \theta_{\mathrm{br}}= & \sqrt{\frac{\varepsilon_{2}}{\varepsilon_{1}}}, \\
\sin \theta_{2,3} & =\sqrt{\frac{\varepsilon_{2} \varepsilon_{\mathrm{tr}}^{4}+\varepsilon_{1} \varepsilon_{\mathrm{tr}}^{4}-2 \varepsilon_{\mathrm{tr}} \varepsilon_{1}^{2} \varepsilon_{2}^{2}}{2 \varepsilon_{1}\left(\varepsilon_{1} \varepsilon_{2}-\varepsilon_{\mathrm{tr}}^{2}\right)\left(\varepsilon_{1} \varepsilon_{2}+\varepsilon_{\mathrm{tr}}^{2}\right)}} \\
& \pm \sqrt{\left(\frac{\varepsilon_{2} \varepsilon_{\mathrm{tr}}^{4}+\varepsilon_{1} \varepsilon_{\mathrm{tr}}^{4}-2 \varepsilon_{\mathrm{tr}} \varepsilon_{1}^{2} \varepsilon_{2}^{2}}{4 \varepsilon_{1}\left(\varepsilon_{1} \varepsilon_{2}-\varepsilon_{\mathrm{tr}}^{2}\right)\left(\varepsilon_{1} \varepsilon_{2}+\varepsilon_{\mathrm{tr}}^{2}\right)}\right)^{2}-\frac{\varepsilon_{2} \varepsilon_{\mathrm{tr}}^{2}}{\varepsilon_{1}\left(\varepsilon_{1} \varepsilon_{2}+\varepsilon_{\mathrm{tr}}^{2}\right)}}
\end{aligned}\right.
$$

с другой стороны, дают возможность рассчитать две из трех диэлектрических проницаемостей сред по установленным экспериментально значениям углов $\theta_{2}$ и $\theta_{3}$. Для примера выпишем выражения для $\varepsilon_{\mathrm{tr}}$ и $\varepsilon_{2}$, приняв значение $\varepsilon_{1}=1$, что соответствует типичной экспериментальной ситуации:

$$
\begin{gathered}
\varepsilon_{\mathrm{tr}}=\frac{\varepsilon_{2}=\operatorname{tg}^{2} \theta_{\mathrm{br}},}{2 \cos \theta_{2} \sqrt{\varepsilon_{2}-\sin ^{2} \theta_{2}}} \\
+\sqrt{\frac{\varepsilon_{2}^{2}}{4\left(\varepsilon_{2}-\sin ^{2} \theta_{2}\right) \cos ^{2} \theta_{2}}-\sin ^{2} \theta_{2} .}
\end{gathered}
$$

Знак плюс в решении квадратного уравнения (9) выбран из условия значения диэлектрической проницаемости больше единицы. 


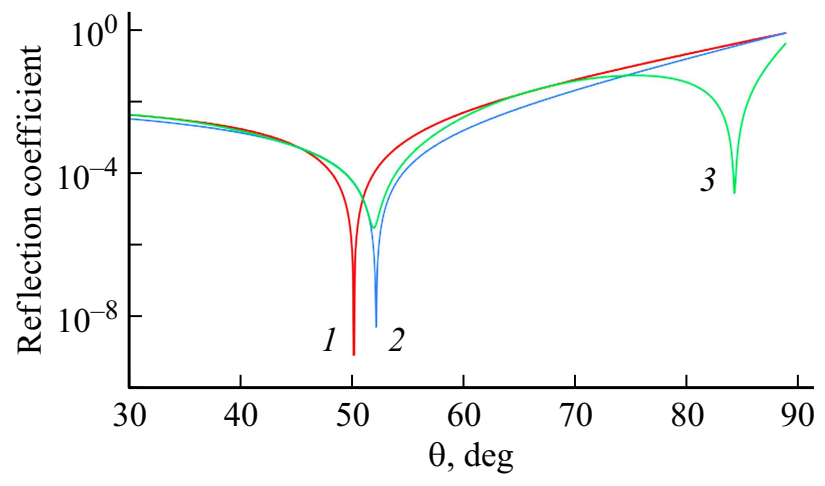

Рис. 4. Зависимость коэффициента отражения от угла падения $\theta$. Расчет выполнен при следующих значениях параметров: $\lambda=532 \mathrm{~nm}, \varepsilon_{1}=2.25, \varepsilon_{2}=3.24, \varepsilon_{\mathrm{tr}}=2.34$. Кривые соответствуют следующим значениям толщины слоя: 0 (1), 137 (2), $397 \mathrm{~nm}(3)$.

Для иллюстрации изложенного на рис. 4 приведена зависимость коэффициента отражения для трехслойной структуры, рассчитанная по формуле (1), где присутствуют три не смещаемых минимума.

Выражение (10) включает особую точку, когда величина

$$
\varepsilon_{1} \varepsilon_{2}-\varepsilon_{\mathrm{tr}}^{2}=0
$$

Непосредственный расчет показывает, что решением уравнения (9) в этом случае является значение $\theta_{2}=0$ при толщине слоя $-\lambda / 4 / \varepsilon_{\mathrm{tr}}$. Соотношение (11) используется в оптике как условие для расчета просветляющих покрытий. Наше рассмотрение показывает, что условие (11) определяет не смещаемый минимум коэффициента отражения. Только в этом случае возможно просветление для пластин произвольной толщины.

Уравнение (9) позволяет определить условие, при котором не смещаемый минимум находится вблизи скользящего угла падения. Подставляя в (9) значение угла падения, равное $\pi / 2$, получаем, что диэлектрическая проницаемость слоя в этом случае должна быть близка диэлектрической проницаемости верхней среды - $\varepsilon_{1}$.

В заключение раздела сформулируем следующие выводы.

1. В общем случае коэффициент отражения плоской монохроматической электромагнитной волны, линейно поляризованной в плоскости падения, от трехслойной структуры имеет три не смещаемых абсолютных минимума, положение которых определяется диэлектрическими проницаемостями подложки, слоя и длиной волны падающего излучения.

2. Ни при каких значениях материальных параметров слоя коэффициент отражения не обращается в нуль при угле падения, соответствующем углу Брюстера слоя. Исключение составляет плоскопараллельная пластина, граничащая со средами, имеющими равные диэлектрические проницаемости.
3. Несмещаемые минимумы могут располагаться во всем диапазоне углов падения, включая нормальное и скользящее.

\section{Инварианты коэффициента отражения}

В настоящем разделе мы акцентируем внимание на альтернативном подходе к решению обратной задачи эллипсометрии, отказавшись от поиска решения уравнения Друде и сосредоточившись на поиске такого набора наблюдаемых параметров, которые однозначно восстанавливают материальные параметры структуры. Суть метода проиллюстрируем геометрией типичной экспериментальной ситуации, при которой трехслойная структура представляет собой плоскопараллельную пластину толщиной $d$ и диэлектрической проницаемостью $\varepsilon_{\mathrm{tr}}$, граничащую с полубесконечными средами с равными диэлектрическими проницаемостями $\varepsilon_{1}$. Как следует из (4), коэффициент отражения для такой геометрии при угле падения $\theta=\theta_{\mathrm{tr}}$ строго обращается в нуль, что, казалось бы, открывает прямой способ определения диэлектрической проницаемости плоскопараллельной пластины, в воздухе. Положение минимума коэффициента отражения в этом случае, во-первых, не зависит от толщины пластины, а во-вторых, однозначно определяет ее диэлектрическую проницаемость:

$$
\varepsilon_{\mathrm{tr}}=\varepsilon_{1} \operatorname{tg}^{2} \theta_{\mathrm{tr}}
$$

Однако, несмотря на схожесть соотношений (5) и (12), они отличаются тем, что соотношение (5) определяет угол Брюстера при отражении от двух полупространств, в то время как соотношение (12) относится к пластине в воздухе. Недостатком такого способа определения диэлектрической проницаемости пластины является существенная зависимость результата от способа обработки поверхностей пластины. Если минимизировать влияние нарушенного обработкой приповерхностного слоя не удается, приходится вводить дополнительный слой с неизвестной толщиной и неизвестным показателем преломления, что резко усложняет задачу. В этом случае приготовление пластины в виде клина не спасает ситуацию. Как мы видели, ни при каких значениях материальных параметров слоя коэффициент отражения не обращается в нуль при угле падения, соответствующем углу Брюстера слоя. Соответственно положение минимума коэффициента отражения в области, близкой к углу Брюстера, не определяется соотношением (5).

Проанализируем ситуацию подробнее. С этой целью определим положение нулей коэффициента отражения при падении плоской монохроматической волны, поляризованной в плоскости падения, на плоскопараллельную пластину под углом $\theta$ в диапазоне $[0-\pi / 2]$. Учитывая, что в данной геометрии $r_{\mathrm{tr} 2}=-r_{1 \mathrm{tr}}$, для 
коэффициента отражения имеем следующее выражение:

$$
\begin{aligned}
R & =|r|^{2}= \\
& =r_{1 \mathrm{tr}}^{2} \frac{2\left[1+r_{1 \mathrm{tr}}^{4}\right]+4 r_{1 \mathrm{tr}}^{2} \cos ^{2} 2 \delta-2\left(1+r_{\mathrm{lt}}^{2}\right)^{2} \cos 2 \delta}{\left[\left(1+r_{1 \mathrm{tr}}^{4}\right)-2 r_{1 \mathrm{tr}}^{2} \cos 2 \delta\right]^{2}} .
\end{aligned}
$$

Равенство нулю $R$ возможно в следующих случаях:

$$
\begin{gathered}
r_{1 \mathrm{tr}}=0, \\
2\left[1+r_{1 \mathrm{tr}}^{4}\right]+4 r_{1 \mathrm{tr}}^{2} \cos ^{2} 2 \delta-2\left(1+r_{1 \mathrm{tr}}^{2}\right)^{2} \cos 2 \delta=0 .
\end{gathered}
$$

Первое из этих уравнений, как и следовало ожидать, определяет не смещаемый минимум, отвечающий углу $\theta_{\text {tr }}$, который в данном случае равен углу Брюстера. Второе уравнение определяет смещаемые минимумы, положение которых зависит от толщины пластины. Оно удовлетворяется при значениях углов падения, соответствующих условию:

$$
\delta=2 \pi \frac{d}{\lambda} \sqrt{\varepsilon_{\mathrm{tr}}-\varepsilon_{1} \sin ^{2} \theta}=\pi n
$$

Отсюда

$$
\sin ^{2} \theta_{n}=\frac{\varepsilon_{\mathrm{tr}}}{\varepsilon_{1}}-\frac{1}{\varepsilon_{1}}\left(\frac{\lambda}{d} \frac{n}{2}\right)^{2}
$$

Область значений $n$, при которых существуют углы падения, удовлетворяющие (16), определяется условиями

$$
0 \leq \frac{\varepsilon_{\mathrm{tr}}}{\varepsilon_{1}}-\frac{1}{\varepsilon_{1}}\left(\frac{\lambda}{d} \frac{n}{2}\right)^{2} \leq 1
$$

Границами диапазона $n_{\max }$ и $n_{\min }$ являются целочисленные решения уравнений

$$
\left\{\begin{array}{l}
\frac{\varepsilon_{\mathrm{tr}}}{\varepsilon_{1}}-\frac{1}{\varepsilon_{1}}\left(\frac{\lambda}{d} \frac{n_{\max }}{2}\right)^{2}=0 \\
\left.\frac{\varepsilon_{\mathrm{tr}}}{\varepsilon_{1}}-\frac{1}{\varepsilon_{1}}\left(\frac{\lambda}{d} \frac{n_{\min }}{2}\right)^{2}=1\right) .
\end{array}\right.
$$

Отсюда находим значение $N$, определяющее количество нулей уравнения (13),

$$
N=n_{\max }-n_{\min }
$$

С учетом минимума, отвечающего углу Брюстера, заключаем, что коэффициент отражения при падении плоской монохроматической волны, поляризованной в плоскости падения на плоскопараллельную пластину, имеет $N+1$ значение углов падения, при которых $R=0$. На рис. 5 приведен график коэффициента отражения от плоскопараллельной структуры, рассчитанный по формуле (13). Жирными точками на оси абсцисс отмечены значения углов, отвечающие нулям коэффициента отражения и рассчитанные по формуле (16), штриховой вертикальной линией обозначен не смещаемый нуль, отвечающий углу Брюстера.

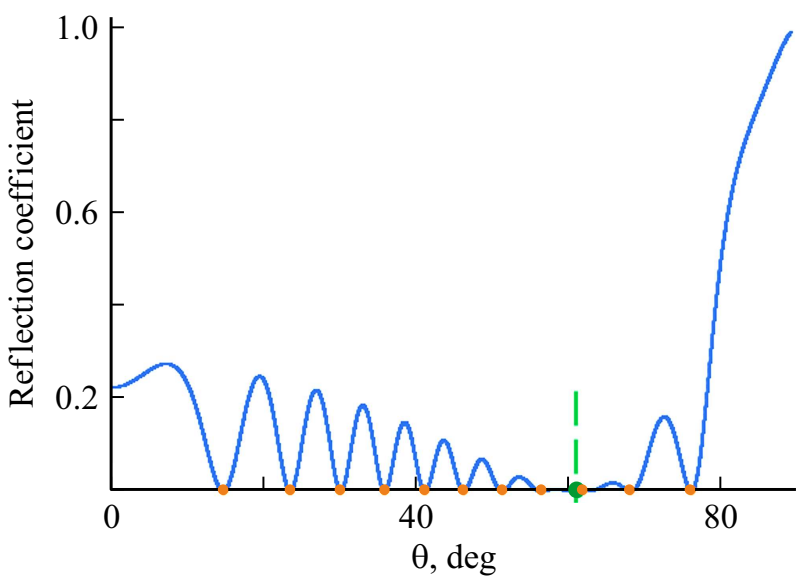

Рис. 5. Зависимость коэффициента отражения от угла падения $\theta$. Расчёт выполнен при следующих значениях параметров: $\lambda=532 \mathrm{~nm}, \varepsilon_{1}=1, \varepsilon_{2}=1, \varepsilon_{\mathrm{tr}}=3.24, d=10 \mu \mathrm{km}$.

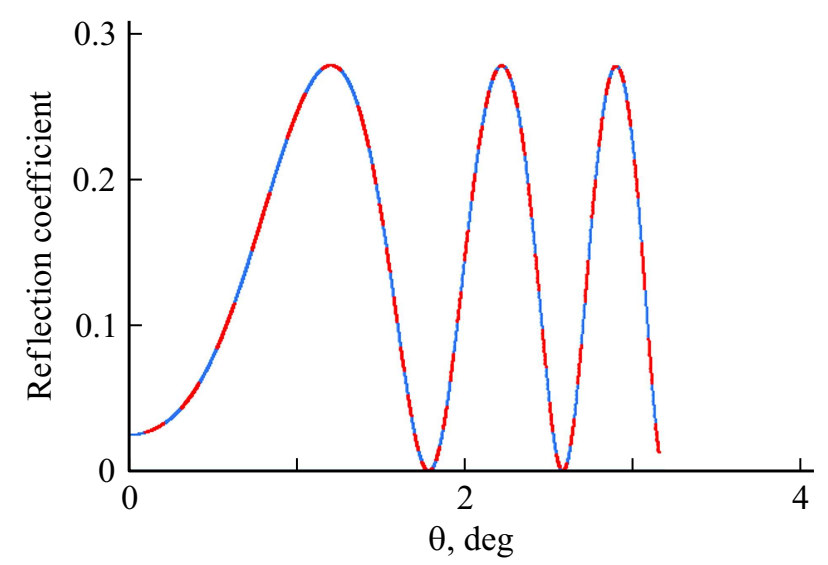

Рис. 6. Угловая зависимость коэффициента отражения. Красная штриховая кривая отражает решение прямой задачи. Синяя штриховая линия обозначает угловую зависимость коэффициента отражения с использованием параметров, рассчитанных при решении обратной задачи - формулы (17),(18). Расчет прямой задачи выполнен при следующих значениях параметров: $\lambda=532 \mathrm{~nm}, \varepsilon_{1}=1, \varepsilon_{2}=1, \varepsilon_{\mathrm{tr}}=2.56, d=1 \mathrm{~mm}$.

Для трех последовательных минимумов имеем

$$
\begin{gathered}
\sin ^{2} \theta_{n}=\frac{\varepsilon_{\mathrm{tr}}}{\varepsilon_{1}}-\frac{1}{\varepsilon_{1}}\left(\frac{\lambda}{d} \frac{n}{2}\right)^{2}, \\
\sin ^{2} \theta_{n+1}=\frac{\varepsilon_{\mathrm{tr}}}{\varepsilon_{1}}-\frac{1}{\varepsilon_{1}}\left(\frac{\lambda}{d} \frac{n+1}{2}\right)^{2}, \\
\sin ^{2} \theta_{n+2}=\frac{\varepsilon_{\mathrm{tr}}}{\varepsilon_{1}}-\frac{1}{\varepsilon_{1}}\left(\frac{\lambda}{d} \frac{n+2}{2}\right)^{2} .
\end{gathered}
$$

Нетрудно видеть, что величина

$$
\text { Inv }_{d}=\sin ^{2} \theta_{n+2}-2 \sin ^{2} \theta_{n+1}+\sin ^{2} \theta_{n}=-\frac{2}{\varepsilon_{1}}\left(\frac{\lambda}{2 d}\right)^{2}
$$

является инвариантом коэффициента отражения для плоскопараллельной пластины, что позволяет записать 
выражение для толщины пластины:

$$
d=\frac{\lambda}{\sqrt{-2 \varepsilon_{1} \operatorname{Inv} v_{d}}}
$$

Немногим сложнее обстоит дело с величиной

$$
I n v_{\varepsilon}=\sin ^{2} \theta_{n}-\frac{\left(4 \sin ^{2} \theta_{n+1}-3 \sin ^{2} \theta_{n}-\sin ^{2} \theta_{n+2}\right)^{2}}{8\left(\sin ^{2} \theta_{n+2}-2 \sin ^{2} \theta_{n+1}+\sin ^{2} \theta_{n}\right)}
$$

которая также является инвариантом, в чем можно убедиться непосредственной подстановкой. Этот инвариант позволяет рассчитать диэлектрическую проницаемость пластины:

$$
\varepsilon_{\mathrm{tr}}=\varepsilon_{1} \operatorname{In} v_{\varepsilon}
$$

Сумма $k$ инвариантов между $n$ и $n+k$ минимумами:

$$
k \operatorname{Inv} v_{d}=\left(\sin ^{2} \theta_{n+1}-\sin ^{2} \theta_{n}\right)-\left(\sin ^{2} \theta_{n+k}-\sin ^{2} \theta_{n+k-1}\right),
$$

позволяет рассчитать значение инварианта по измерению разницы в положении двух пар минимумов, отстоящих друг от друга на $k$ значений. С экспериментальной точки зрения, это должно повысить точность определения $\operatorname{Inv}{ }_{d}$.

Соотношения (17),(18) решают обратную задачу эллипсометрии - задачу определения материальных параметров пластины (диэлектрической проницаемости и толщины) по измерению положения трех последовательных минимумов коэффициента отражения плоской монохроматической электромагнитной волны, поляризованной в плоскости падения. Эти углы целесообразно выбрать в качестве наблюдаемых параметров. Подчеркнем, что вывод соотношений $(17),(18)$ основан только на анализе соотношения (15), определяющем фазовую толщину слоя $\delta$.

Рисунок 6 демонстрирует результат численного эксперимента, при котором на первом этапе был проведен расчет коэффициента отражения по формуле (13) синяя штриховая кривая. На втором этапе были рассчитаны материальные параметры структуры по формулам (17),(18) для трех последовательных углов падения. На третьем этапе вновь был проведен расчет коэффициента отражения по формуле (13) с использованием значений параметров, полученных на втором этапе красная штриховая кривая.

\section{Инварианты коэффрициента отражения при наличии пленки на поверхности плоскопараллельной пластины}

Можно предположить, что наличие пленки, толщина $\Delta d$ которой много меньше толщины пластины, а диэлектрическая проницаемость $\varepsilon_{h}$ мало отличается от диэлектрической проницаемости пластины:

$$
\begin{gathered}
\Delta d \ll d, \\
\Delta \varepsilon_{\mathrm{tr}}-\varepsilon_{h} \ll \varepsilon_{\mathrm{tr}},
\end{gathered}
$$

вносит слабые изменения в значения инвариантов. При этих условиях их можно разложить по этим малым параметрам. Для дифференциалов будем иметь

$$
\left\{\begin{array}{l}
\Delta I n v_{d}=-\frac{1}{\varepsilon_{1}}\left(\frac{\lambda}{d}\right)^{2} \frac{\Delta d}{d} \\
\Delta I n v_{\varepsilon}=\frac{\Delta \varepsilon}{\varepsilon_{1}}
\end{array}\right.
$$

Таким образом, измеряя значения инвариантов до и после нанесения плёнки, можно получить значения толщины и диэлектрической проницаемости плёнки:

$$
\begin{gathered}
\Delta d=-\varepsilon_{1} d\left(\frac{d}{\lambda}\right)^{2} \Delta I n v_{d}, \\
\varepsilon_{h}=\varepsilon_{\mathrm{tr}}-\varepsilon_{1} \Delta \operatorname{In} v_{\varepsilon}
\end{gathered}
$$

В том случае, когда параметры пластины известны, значения ее инвариантов можно изначально рассчитать.

\section{Заключение}

Предложен альтернативный метод решения обратной задачи эллипсометрии, основанный исключительно на анализе фазовых соотношений отраженных от структуры волн. Показано существование двух инвариантов коэффициента отражения для плоскопараллельной пластины. Предложен набор трех наблюдаемых параметров, не используемых ранее, позволяющий однозначно восстановить материальный параметры структуры по полученным аналитическим выражениям.

\section{Финансирование работы}

Работа выполнена при финансовой поддержке Министерства науки и высшего образования Российской Федерации, проект No.RFMEFI58117X0026.

\section{Конфликт интересов}

Авторы заявляют, что у них нет конфликта интересов.

\section{Список литературы}

[1] Ефимов А.М., Постников Е.С. Физические основы и формализм оптики и спектроскопии оптических материалов. СПб: Университет ИТМО, 2015.

[2] Polovinkin V.G., Svitasheva S.N. // Optoelectronics Instrumentation Data Processing. 1999. V. 4. P. 79.

[3] Dultsev F.N., Kolosovsky E.A. // Advances in Condensed Matter Physics. 2015. vol. 2015. p. 1-8.

[4] Швец, B.A., Спесивцев Е.В. Эллипсометрия. Новосибирск, 2013.

[5] Зинченко С.П., Ковтун А.П., Толмачев Г.Н. // ЖТФ. 2009. T. 79. B. 11. C. 128.

[6] Шагаев В.В. // ЖТФ. 2015. Т. 85. В. 12. С. 6.

[7] Сивухин Д.В. Общий курс физики. Оптика. М.: Наука, 1985. 